\title{
Immunoinformatics guided design of a universal epitope-based vaccine against Kaposi Sarcoma
}

Hammadul Hoque ( $\square$ hammadul-geb@sust.edu )

Shahjalal Unversity of science and Technology, Sylhet

Sumaiya

Shahjalal Unversity of science and Technology, Sylhet

Mahmudul Hasan

Shahjalal Unversity of science and Technology, Sylhet

Md. Mashiur Rahaman

Shahjalal Unversity of science and Technology, Sylhet https://orcid.org/0000-0002-5089-8707

G. M. Nurnabi Azad Jewel

Shahjalal Unversity of science and Technology, Sylhet

\section{Research Article}

Keywords: Immunoinformatics, Kaposi Sarcoma, Virus, Molecular Docking, Molecular Dynamics Simulation, Multi-epitope Vaccine

Posted Date: January 28th, 2022

DOI: https://doi.org/10.21203/rs.3.rs-1307300/v1

License: (a) (i) This work is licensed under a Creative Commons Attribution 4.0 International License. Read Full License 


\section{Abstract}

The Kaposi sarcoma-associated herpesvirus (KSHV) is a virus that is identified as a direct carcinogen, causes Kaposi sarcoma, primary effusion lymphoma and multicentric Castleman disease. Despite this, there is no permanent treatment. This work intended to develop a multi-epitope vaccine aiming KSHV's key glycoproteins involved in viral entry. After applying rigorous immunoinformatics study and numerous immunological filters, the multi epitope vaccine was created, comprising of potent CTL, HTL and BCL epitope. A series of computational evaluations established the general dependability of the putative vaccine, and a molecular dynamics simulation established the vaccine's overall stability. Docking experiments revealed that the vaccination can make stable interactions with Toll-Like Receptors. Codon optimization and insertion into the cloning vector revealed that the vaccine could be expressed proficiently in the $E$. coli expression system. Finally, an immune simulation was done to assess the vaccine's potency to trigger an immune response.

\section{Introduction}

The Kaposi sarcoma (KS) is the soft tissue tumor that previously gained widespread attention as an AIDS-defining disease [1], this human malignancy is also linked with other medical conditions such as multicentric Castleman's disease (MCD), inflammatory cytokine syndrome (KICS), primary effusion lymphoma (PEL), and immunosuppression regarding an organ transplant [2]. The virus Kaposi sarcoma-associated herpesvirus (KSHV) is certainly the causative agent of Kaposi sarcoma malignancy, it is formerly known as Human Herpesvirus-8 (HHV-8). HHV-8 is also recognized as a human carcinogen, according to Global cancer statistics 2018, it is responsible for about 40,000 cancer cases and 20,000 deaths worldwide each year [3], and especially the AIDS patients have a 1,00,000-fold increased risk of contracting KS than the general population [4]. As the risk of KS malignancy cases linked with HIV and lymphoproliferative disorders are not only significantly common, but these malignancies can also occur in the absence of these coinfections in any immune-compromised, malnourished, or solid organ transplant recipient infected with HHV-8 [5].

KSHV is a gammaherpesvirus with a double-stranded DNA virus. This is a lymphotropic virus that encounters lytic replication in epithelial cells comprising skin, blood vessels, and organs. These viruses can remain dormant in their host cells for an indefinite period of time. HHV8/KSHV has seven recognized subtypes (A, B, C, D, E, F, and Z) [6]. The HHV-8 genome is around $170 \mathrm{~kb}$ in size and encodes about 81 ORFs [7]. HHV-8 encompasses multiple viral proteins that are crucial for infecting, establishing latency, and cancer development, including the major latency antigen latency-associated Nuclear Antigen-1 (LANA), Kaposins are latent proteins that play a critical role in tumor formation, viral IL-6 (vIL-6), Rta protein (ORF50), viral cyclin (v-cyclin), viral IL-6 (vIL-6) and viral Fas ligand inhibitory protein (v-FLIP) [8]. Human herpesvirus-8 is typically transmitted sexually, and infection occurs rarely before puberty [9]. In Uganda, seropositivity to HHV-8 was closely linked with the frequency of transfusions, with a calculated risk of infection of 2.6 percent per transfused blood component [10].

The treatment for standard Kaposi sarcoma is retroviral therapy. However, not all cases of KS cancer are identical, neither will all patients react to the same treatment. So, to decrease the development of Kaposi sarcoma in all HHV-8 positive individuals a targeted antiviral therapy is needed. Study found that antiviral treatment with the ganciclovir and foscarnet family of medications that are powerful inhibitors of herpesvirus DNA polymerase, was efficacious in reducing KS lesions in HIV affected and non-affected patients but not in all cases [11]. Martín-Carbonero et al; (2004); demonstrated that intravenous pegylated liposomal doxorubicin is more effective than highly active antiretroviral treatment (HAART) alone in treating AIDS-KS patients [12]. However, other studies have found that pegylated liposomal therapies such as Doxil and Paclitaxel are effective against KS but also have a high rate of toxicity [13]. Additionally, intravenous liposomal tretinoin treatment in AIDS-KS patients exhibited a number of side effects and a partial response to Kaposi sarcoma lesions [14]. In terms of Transplant patients with KS respond to immune reconstitution, consequently decreasing the immunosuppressive dosage enhances graft rejection. In this event rapamycin predominantly inhibits tumor growth, resulting in stable condition rather than tumor regression $[15,16]$.

Vaccinations are the most appropriate method to prevent viral infections, and multi-epitope vaccines offer a broader immune response spectrum than single-epitope vaccines. HHV-8 vaccination has not yet been documented. Immune responses are one of the most critical aspects in fighting tumor progression in Kaposi sarcoma patients. Traditional vaccine design approaches include the addition of excess antigenic load and raises the risk of poor immunogenicity also expense [17]. The use of peptide-based vaccines, which have highly specific immune responses, can overcome these limitations. Progress in computational biology aid in designing an active vaccine synthesis [18].

An ideal strategy for the prevention and treatment of viral infections or tumor, a multi-epitope vaccine capable of activating both humoral and adaptive immune responses [19]. Numerous in silico and immunoinformatics techniques for the prediction of antigenic epitopes have been developed, with potential translational applications, these vaccinomics approaches are used to construct a multi-epitope vaccine against HHV-8 [20]. To strengthen the immune response against HHV-8, the multi-epitope vaccine should trigger Cytotoxic T lymphocytes (CTL), Helper T lymphocytes (HTL), induce cytokine production and create long lasting memory. 


\section{Materials And Methods}

\subsection{Selection of Human Herpesvirus-8 strain and protein sequence retrieval}

As a repository for biomedical and genetic data, the server National Center for Biotechnology Information (NCBI) [21] makes significant contributions to research and health. This was used to identify and analyze Human Herpesvirus-8 strains in addition to related data such as genus, family, transmission, host, illness, genome, and proteome. UniProtKB [22] contains a lot of biological information about proteins. The database had five types of the envelope glycoprotein sequence of the Human herpesvirus 8 type P strain that had already been reviewed. A flow chart that shows how immunoinformatics techniques can be used to make a multiepitope-based chimera vaccine has been shown below (Figure 1).

\subsection{Screening of antigenic proteins}

The antigenicity of a protein was determined using the VaxiJen v2.0 tool [23]. A thorough investigation has been conducted on the antigenic and physiochemical characteristics of the sequences that were discovered. Proteins that were antigenic and had the appropriate characteristics were carefully chosen for further investigation.

\subsection{T cell epitopes identification}

It was necessary to utilize the NetCTL 1.2 server [24] in order to identify cytotoxic T lymphocyte (CTL) epitopes for the proteins of Human Herpesvirus- 8 that were chosen. There is a sensitivity and specificity range of $54-89 \%$ and $94-99 \%$ respectively, for the epitope predicted by the server. CTL epitopes were identified in this work using twelve MHC class I supertypes, which were utilized in the search for CTL epitopes. They received scores of 0.05 for TAP transport efficiency, 0.15 for $C$ terminal cleavage, and 0.75 for the epitope identification threshold, accordingly. It was only recently that such an approach was devised for class I immunogenicity using 9-mer peptides [25]. The epitope identification tool TepiTool [26] was used to discover 15-mer helper T lymphocyte (HTL) epitopes. The IEDB suggested 27 HLA class II reference alleles for MHC class II alleles [27] in order to identify MHC class II alleles. It was used to predict HTL epitopes based on the IC50 value, which was set to less than or equal to $250 \mathrm{nM}$ by default, which was used to predict HTL epitopes.

\subsection{Linear B cell epitope identification}

B cell lymphocyte $(\mathrm{BCL})$ epitopes are critical components of the immune system that trigger an adaptive immunological response in the body. They have the potential to be employed as vaccine building blocks because they stimulate humoral immunity via the secretion of antigen-neutralizing Immunoglobulins. Linear and conformational receptors are the two kinds of surface receptors for B-cell epitopes that could be found on B cells' surfaces. The BCPred server was used to predict linear B-cell epitopes with a specificity of 75\% for 20-mer linear Bcell epitopes. A total of 10 different epitope sequences for glycoproteins were predicted [28].

\subsection{Selection of anticipated epitopes for further study}

\subsubsection{Prediction of B and T cell epitope's toxicity, antigenicity, allergenicity, transmembrane and immunogenicity topology}

For the purpose of selecting the most appropriate B and T-cell epitopes, the toxicity, allergenicity and antigenicity of previously predicted $\mathrm{BCL}$, CTL and HTL epitopes were examined. Unlike previous versions of the software, VaxiJen v2.0 categorizes antigens purely on the basis of protein physicochemical features rather than on sequence alignment. As the target organism and a default threshold value were both utilized, we were able to predict the epitopes that would be most effective. For the purpose of estimating the allergenicity of our putative epitopes, we utilized the AllerTOP v2.0 server [29]. It was only employed for the non-allergen epitopes that needed to be assessed for toxicity, so ToxinPred was used. It is possible to determine epitopes' cytotoxic potential by using the ToxinPred database [30], which also contains information on other essential physicochemical features. The transmembrane helices in epitopes were highlighted using the TMHMM v2.0 program. This approach was used to determine whether a certain epitope was located inside or outside the helices [31]. In the end, the immunogenicity of the epitopes was analyzed by the use of the IEDB server [32].

\subsubsection{Prediction of HTL epitopes induced by IFN-, IL-4, and IL-10}

Immune cytokines (interferon-y [IFN-ү], interleukin-4 [IL-4], and interleukin-10 [IL-10]), which stimulate macrophages, dendritic cells, and natural killer cells in response to viruses, bacteria, and parasites. Those are vital cytokines in the regulation of both innate and adaptive immune responses. Not all HTL epitopes have the ability to induce cytokine production, however. It follows that including HTL epitopes into the vaccine is a key step in increasing the potency of the vaccine. As a consequence, the IFNepitope server [33] was utilized to discover HTL epitopes that triggered IFN production. In accordance with the Motif and SVM approaches, the server predicts epitopes that induce IFN 
production.IL4pred server [34] and IL10pred server [35] are two web-based prediction servers that are used to forecast cytokines IL-4 and IL10, respectively, in humans. Both programs used the SVM-based default technique.

\subsubsection{Population coverage and conservancy analysis of the epitopes}

Due of the vast differences in immune profiles amongst populations, the multi-epitope vaccine should be efficient in combating the virus. As a result, the population coverage of the specified CTL epitopes was determined using the Population Coverage tool on the IEDB website [36].

Also, The conservancy analysis tool of IEDB was also used to identify the most conserved CTL epitopes across strains [37]. The conservancy was determined by focusing on the antigenic protein's identity. Epitopes with $100 \%$ conservation were chosen for vaccine development.

\subsection{Construction of multi-epitope vaccine candidate}

The epitopes that matched all of the parameters were utilized to build the vaccine design that was ultimately selected. Beginning with a Methionine at the Carboxy-terminal end of the recommended vaccine, which is then linked to a $\beta$ defensin-2 adjuvant [38] (a TLR4 agonist) by an EAAAK linker. When used in conjunction with a vaccine, adjuvants may boost its immunogenicity. A short peptide (RKKRRQRRR) derived from the HIV-TAT protein [39] was inserted immediately after the adjuvant, which may improve cell penetration and facilitate in MHC class I processing. GGGS linkers were distributed throughout the chimera sequence in order to provide 3D structural stability. Conjugation of CTL epitopes is accomplished by the inclusion of an AAY linker in the contract [20]. In order to aid in the cellular processing of the vaccine, the unique linkers AAY and HEYGAEALERAG, which function as cleavage sites for the proteasomal and lysosomal systems, respectively, were inserted [40-42]. Another adjuvant RS-09 [43] was added after the last epitope to further boost immunity even more.

\subsection{Analysis of immunogenic and physicochemical characteristics}

A series of immunogenicity tests were performed using the VaxiJen 2.0, AllerTop 2.0, and ToxinPred servers in order to validate the immunogenic properties (antigenicity, allergenicity, and toxicity) of the newly proposed vaccine. Expasy's ProtParam tool was used to display the vaccine's functional characteristics based on the vaccine's molecular weight, aliphatic index, pl, hydropathicity, instability index, GRAVY values, and predicted half-life in E. coli. It is possible that insufficient protein solubility may prevent therapeutic protein expression in E. coli, and it is most probable that this will be the most common reason for insufficient protein production, which would significantly increase the expense of experimental research. To analyze the solubility of the designed vaccine, the researchers employed SoluProt 1.0 , a freely available web server [44].

\subsection{Prediction of the secondary structure}

For the secondary structure prediction of the proposed vaccine, PSIPRED 4.0 was utilized [45]. With the vaccine sequence as a guide, it predicts the formation of the helix, sheet, and coil structures in the vaccine candidate sequence.

\subsection{Prediction of the tertiary structure and refinement of the vaccine}

A 3D structure of the intended vaccine was created with the aid of the Robetta web server [46], which was necessary due to a lack of predesigned structures. RoseTTAFold made use of deep learning to correctly predict the three-dimensional structure of every input sequence that was given to it. Robetta created five models on the basis of amino acid sequence that was provided. The model with the lowest estimated error was selected for examination, and it was further refined using the GalaxyRefine option on the GalaxyWEB server [47] to get the lowest possible error estimate. GalaxyRefine runs MD simulations on the structure in order to continually perturb it and subsequently relax it, as seen in the following figure: It was decided which model will be used from five updated models based on the lowest MolProbity Clash score. The original and updated structures were structurally validated using the SAVES v6.0 webserver's ERRAT [48] and PROCHECK [49] options. PROCHECK contains a Ramachandran plot, which allows for the straightforward presentation of residues in the most favorable, considerably acceptable, widely permitted, and disallowed regions in order to evaluate the validity of the vaccine model. Further, with the aid of the ProSA webserver, it was possible to evaluate the likelihood of structural and modeling mistakes in the refined mode [50].

\subsection{Docking with the TLR4 receptor}

The proposed vaccine's affinity for TLR4 was evaluated via the use of molecular docking. The three-dimensional structure of TLR4 (PDB ID: 4G8A) Downloaded from the RCSB PDB site [51] and prepared for docking using the Discovery studio software. In order to dock with the vaccine, only the "A" Chain of TLR4 was preserved. Docking was achieved via the use of the online docking server ClusPro 2.0 [52]. ClusPro constructs docking complexes in three steps: rigid body docking using billions of conformational data, grouping the 1000 lowest energy structures by RMSD, and refining the selected structures using energy reduction. A second docking server, Hawkdock [53] was used for docking and MMGBSA analysis to further increase the confidence in the studies. The complex with the highest ranking was selected, and the subsequent protein-protein interaction of the docked complex was analyzed using another web-based resource, PDBsum [54]. 
Molecular Dynamics simulation is a great computational tool for studying a protein's structural stability at the atomic level [55]. In this study, we used the GROMACS [56] software package (version 2021.04) to conduct an MD simulation of our constructed vaccine using the final pdb file. For this, first, the protein was solvated in a cubic box with SPC/E water molecule using the CHARMM36 force field [57]. The whole system was optimized by using proper periodic boundary conditions and neutralized by adding 3 chloride ions. The entire system was then equilibrated using the steepest descent approach with 5000 iterations for $1 \mathrm{~ns}$ using canonical ensembles (NVT and NPT). Finally, the MD simulation was executed for 100ns by maintaining Berendsen thermostat [58] with no pressure coupling at a constant 300K temperature and 1 atm pressure. The last step of MD simulation includes the analysis RMSD (Root Mean Square Deviation), RMSF (Root Mean Square Fluctuation) and $\mathrm{Rg}$ (Radius of gyration) of the protein from the simulation trajectory.

\subsection{Immune simulation}

To further validate the immunogenicity and immune reaction status of the proposed vaccine, in silico immune simulations were executed using the C-ImmSim server [59]. C-ImmSim is an agent-based method that predicts immune epitopes by using a position-specific scoring matrix (PSSM) and immune interactions by using machine learning strategies. This server also simulates the three vital functional elements of the mammalian system: bone marrow, lymph node, and thymus [59]. In this study, Random seed was set to 12345 while simulation volume and simulation step were set to 10 and 1050, respectively. Each of the three injections was given at a four-week interval, with the time steps of 1,84 , and 168 . The remaining parameters were kept at their default settings [60].

\subsection{Codon optimization for in-silico cloning of the constructed vaccine}

The procedure of reverse translation and adaptation of multi-epitope vaccine codons was carried out using the Java Codon Adaptation Tool (JCat) [61] in order to maximize vaccine production in a suitable host organism. The host organism for the projected vaccine candidate's expression was determined by E. coli K12 strain in this study. In addition, the server response contains a codon adaptation index (CAI), the CDNA sequence, and the ratio of GC content. Finally, using the SnapGene software, the EcoRI restriction enzyme was introduced to the $5^{\prime}$ end of the vaccine nucleotide sequences as an initial cleavage site, and BamHI was added to the 3 ' end of the vaccine nucleotide sequences, and the resulting cloning vector pET-28 (+) was used to incorporate the vaccine nucleotide sequences.

\section{Results}

\subsection{Protein sequence retrieval and estimate of antigenicity}

The five Envelope glycoproteins ( $\mathrm{gB}, \mathrm{gM}, \mathrm{gN}, \mathrm{gH}, \mathrm{gL}$ ) of the Human Herpesvirus 8 type P (isolate GK18, strain HHV-8) were extracted from UniProtKB and used to predict $\mathrm{B}$ and T-cell epitopes in order to create multi-epitope vaccine sequences. The structural proteins were predicted to be highly antigenic by VaxiJen server, with antigenicity values of $0.4483,0.5387,0.5099,0.5391$, and 0.6528 respectively, all exceeding the default threshold value of 0.4 .

\subsection{Cytotoxic and Helper T-cell epitopes prediction}

\subsubsection{Potential cytotoxic T lymphocyte epitopes}

Each cytotoxic T lymphocyte (CTL) epitope was 9 amino acids in length, and a total of 1055 distinct CTL epitopes were listed utilizing the Glycoprotein sequences through the NetCTL v1.2 platform. Among these CTL epitopes, 19 were found highly antigenic, non-allergic, nontoxic, show outside topology, and immunogenic. Based on $100 \%$ conservation and binding affinity of epitopes with MHC-I alleles, a total of ten CTL epitopes (Table 1) were selected among those potential candidates. 
Table 1

Final epitopes of CTL for multi-epitope based vaccine formation

\begin{tabular}{|llllllllll|}
\hline Protein & Epitope & Position & $\begin{array}{l}\text { NetCTL } \\
\text { Score }\end{array}$ & Antigenicity & Immunogenicity & Allergenicity & Toxicity & Topology & Conservancy \\
\hline gB & IVIAIILII & 741 & 0.8967 & 0.5861 & 0.40585 & NA & NT & Outside & $100 \%$ \\
\hline gB & LATLGTVIL & 7 & 0.8898 & 0.4536 & 0.18358 & NA & NT & Outside & $100 \%$ \\
\hline gH & MPTFTTVGY & 356 & 0.9664 & 1.1929 & 0.25938 & NA & NT & Outside & $100 \%$ \\
\hline gH & VPLPHVTYI & 569 & 1.0572 & 0.6850 & 0.0862 & NA & NT & Outside & $100 \%$ \\
\hline gL & FLVAVVRRL & 83 & 1.1278 & 0.5869 & 0.20575 & NA & NT & Outside & $100 \%$ \\
\hline gL & FAVHSIHFA & 41 & 0.8187 & 1.2734 & 0.10583 & NA & NT & Outside & $100 \%$ \\
\hline gM & KLLFVAVIM & 16 & 0.7659 & 0.5995 & 0.30383 & NA & NT & Outside & $100 \%$ \\
\hline gM & LALTVVWYI & 249 & 0.9807 & 0.9868 & 0.2993 & NA & NT & Outside & $100 \%$ \\
\hline gN & ATFFYLVYL & 90 & 1.1841 & 0.6524 & 0.17444 & NA & NT & Outside & $100 \%$ \\
\hline gN & TASTVALAL & 2 & 1.176 & 0.7568 & 0.07589 & NA & NT & Outside & $100 \%$ \\
\hline
\end{tabular}

\subsubsection{Prediction of helper T lymphocyte epitopes}

A total of 1489 epitopes were identified in the Glycoproteins, but only 7 distinct (15-mers) epitopes for helper T lymphocyte (HTL) were extracted from the Glycoproteins sequences (Table 2). In addition to other criteria, they were chosen for their low IC50 value (250 nM), antigenicity, non-allergenicity, non-toxicity, outer topology, IFN- $y$ positivity, and ability to induce HTL epitopes in response to interleukin-4 [IL-4] and interleukin-10 [IL-10] cytokines.

Table 2

Final epitopes of HTL for multi-epitope based vaccine formation

\begin{tabular}{|llllllllll|}
\hline Protein & Position & Epitope & IC50 & Antigenicity & Allergenicity & Toxicity & IFN-y & IL-4 & IL-10 \\
\hline gM & $149-163$ & KHVLMAAFVYCIHFC & 110.28 & 0.4872 & NA & NT & Positive & Inducer & Inducer \\
\hline gL & $37-51$ & LPLFFAVHSIHFADP & 9.22 & 0.9577 & NA & NT & Positive & Inducer & Inducer \\
\hline gH & $503-517$ & FLGLRYDLHPAKLRA & 31.09 & 1.0503 & NA & NT & Positive & Inducer & Inducer \\
\hline gH & $495-509$ & IYRAFSPCFLGLRYD & 39.44 & 1.1001 & NA & NT & Positive & Inducer & Inducer \\
\hline gH & $302-316$ & AQEAAPDIFLVLFQM & 136.4 & 0.4019 & NA & NT & Positive & Inducer & Inducer \\
\hline gB & $14-28$ & ILLVCFCAGAAHSRG & 41.74 & 0.73 & NA & NT & Positive & Inducer & Inducer \\
\hline gB & $205-219$ & AEPGWFPGIYRVRTT & 234.94 & 0.7156 & NA & NT & Positive & Inducer & Inducer \\
\hline
\end{tabular}

\subsection{Prediction of line B-lymphocyte epitopes}

According to preliminary findings, there were a total of 31 epitopes for B-cell lymphocytes $(B C L)$, with just one of them meeting the requirements, which included being antigenic, non-allergenic, and nontoxic. However, just one B-cell epitope (Table 3) was utilized in the vaccine composition, and it was also identified in the vaccine composition's outer topology as well.

Table 3

Final epitopes of BCL for multi-epitope based vaccine formation

\begin{tabular}{|llllllll|}
\hline Protein & Position & Epitope & Score & Antigenicity & Allergenicity & Toxicity & Topology \\
\hline gN & $43-62$ & SARPSPGPTSVTTPGFYDVA & 1 & 0.8762 & NA & NT & Outside \\
\hline
\end{tabular}

\subsection{Population coverage and conservancy analysis of epitopes}

A universal vaccination with a wide range of coverage needs epitopes that are conserved across several strains. It was decided to utilize the IEDB Epitope Conservancy Analysis tool to evaluate epitopes across different strains of Human herpesvirus 8 type P with a sequence identity threshold of $100 \%$. To construct a vaccine, only CTL epitopes with $100 \%$ conservation were considered for inclusion. It was necessary to use the IEDB MHC-I Binding Prediction tool to find the alleles that would be most likely to bind with each predicted CTL epitope in order to assess the population encompassed by those epitopes. 
The IEDB MHC-I Binding Prediction tool was used to find the alleles that may optimally associate with each anticipated CTL epitope. They had the best coverage for Europe (99.45\%) and the lowest for Central Africa (85.24\%). Other large areas got over $90 \%$ coverage (Figure 2 ).

\subsection{Construction of multi-epitope vaccine sequence}

The developed vaccine includes 18 of the most effective epitopes from three classes; $10 \mathrm{CTL}, 7 \mathrm{HTL}$, and $1 \mathrm{~B}$-cell selected from a pool of predicted epitopes. The CTL, HTL, and B-cell epitopes were connected together using the EAAAK, GGGS, and AAY linkers. With the goal of boosting immunogenicity, the vaccine sequence was supplemented with the human $\beta$ defensin-2 adjuvant, the PADRE sequence, the TAT peptide, and the one-of-a-kind linker "HEYGAEALERAG," among other components. Ultimate length of the vaccine was found to be 402aa (Figure 3).

\subsection{Physiochemical properties, antigenicity, allergenicity, toxicity, solubility prediction of vaccine candidate}

According to the results of the ProtParam study (Table 4), the estimated molecular weight of the vaccine candidate was $42990.32 \mathrm{Da}(43$ $\mathrm{kDa})$ and the isoelectric point was $9.73 \mathrm{Da}(43 \mathrm{kDa})$. There are a total of 18 negative amino acid residues (Asp + Glu) and 43 positive amino acid residues (Arg + Lys) in this polypeptide. A number of additional criteria were taken into account, including the aliphatic index and the grand average of hydropathicity (GRAVY) value, both of which were determined to be 89.28 and 0.267 , respectively, and were found to be significant. Moreover, along with the physicochemical features of the constructs, immunological efficiency also has been evaluated. There was an antigenicity score of 0.5359 (antigenic) for the suggested sequences, as well as non-allergenicity and a solubility score of 0.768 for the proposed vaccine sequences.

Table 4

Physicochemical features and antigenicity, allergenicity, toxicity of the vaccine

\begin{tabular}{|ll|}
\hline Properties & Assessment \\
\hline Number of amino acids & 402 \\
\hline Molecular Weight & $42 \mathrm{kDa}$ \\
\hline Theoretical pl & 9.73 \\
\hline Atomic composition & $\mathrm{C}_{1985} \mathrm{H}_{3048} \mathrm{~N}_{540} \mathrm{O}_{500} \mathrm{~S}_{16}$ \\
\hline Estimated half-life (in E. coli) & $>10$ hours \\
\hline Aliphatic index & 89.28 \\
\hline Instability index & 36.20 (Stable) \\
\hline Grand average of hydropathicity (GRAVY) & 0.267 \\
\hline Antigenicity & 0.5359 \\
\hline Allergenicity & Non-allergen \\
\hline Toxicity & Non-toxic \\
\hline Solubility & 0.768 (Soluble) \\
\hline
\end{tabular}

\subsection{Prediction of secondary structure}

The $\alpha$-helix, $\beta$-strand, and random coil characteristics of the final vaccine candidate were analyzed using the PSISPRED server. On the server, it was determined that the vaccine had $46.52 \%$ of the residues that were in the a-helix conformation, $13.93 \%$ that were in the $\beta$-strand conformation, and $39.55 \%$ that were in the coil structure conformation. Figure 4 shows the structural properties in the alpha-helix, betastrand, and random coils of the protein.

\subsection{Tertiary structure, refinement, and validation analysis of the final candidate vaccine}

Robetta generated five 3D structural models for the supplied protein sequence. The first predicted model (Figure 5) was chosen for further investigation since it had the lowest estimated error rate. The GalaxyRefine was also employed to improve the previous 3D model obtained 
from Robetta. After the refining process, the fraction of residues within the ideal region has increased, resulting in five better models being produced. From there, the third model that featured the lowest MolProbity and Clash scores were retrieved as the chosen vaccine model because it was relatively better than all other models. To validate the refined 3D structure, the Procheck tool in SAVES.v6 server was used. Detailed analysis of the Ramachandran plot of the final modeled vaccine illustrated that the protein has residues of $94.8 \%$ in the most favorable areas, $3.7 \%$ in the additional allowed areas, $0.9 \%$ in generously allowed areas, and $0.6 \%$ in disallowed areas. Each value is far better than the Ramachandran plot evaluation of the initial model, which has $84.5 \%$ in favorable areas, and $14.1 \%$ in allowed areas.

\subsection{Molecular docking of refined protein with immune receptor TLR-4}

To analyze the binding interactions between the constructed vaccine and TLR4, docking simulation was done through the online ClusPro docking server. Cluspro produced 29 clusters for the docked complex. Cluster 0 had the lowest energy score (-1424.5) and was selected for further evaluation. PDBsum analysis (Figure 6) showed that the vaccine-TLR4 complex formed 18 hydrogen bonds, 6 salt-bridges, and 193 non-bond interactions. MMGBSA Analysis from HawkDock showed the free binding energy to be -67.02 (kcal/mol) which is again an indication of stable complex formation.

\subsection{Molecular dynamics analysis of final vaccine candidate}

The simulation trajectory was used to calculate the RMSD, RMSF values of the vaccine. RMSD is used to examine whether or not a protein is stable by computing the Deviations from its beginning position to its final conformation. The RMSD analysis depicted in the Figure shows initial fluctuations of the protein till 20ns of the simulation, after that the protein reached an equilibration. The overall RMSD value (Figure 7) of the vaccine stayed within the $0.8-1.2 \mathrm{~nm}$ region without any extreme fluctuations throughout the simulation. This indicates the protein to be structurally stable during the simulation. RMSF analysis from the simulation can help to explain the average fluctuation of amino acids over time. The RMSF values (Figure 8) again remained consistent throughout the simulation, with no unexpected fluctuations. The RMSF values stayed between $0.2 \mathrm{~nm}$ to $0.5 \mathrm{~nm}$ throughout the simulation indicating the absence of any unwanted extreme flexible regions.

\subsection{In silico immune response simulation}

Results from C-ImmSim showed that the immune response in the second and third reactions was relatively high compared to the first dose of the vaccine as expected. Increased numbers of Natural Killer cells and Macrophages were observed throughout the simulated time period (Figure 9A). Antigen concentrations were reported to be high during the primary immune stimulation responses.

There was a comparable increase in T-cytotoxic cell and Th cell reactions with the formation of memory (Figure 9B). Based on Figure 9B, there was a considerable upsurge in immunoglobulin activities $(\lg M+\lg G, \lg M, \lg G 1+\lg G 2)$ with rapid antigen clearance. A significant increase in the population of B-cells, including memory B-cells after vaccination, suggests the possibility of isotype switching and the formation of long-term memory and long-lasting immune response. IFN-g, TGF-b, IL-10, and IL-12 levels were also substantially higher in secondary and tertiary responses than in primary ones, indicating a strong immune response.

\subsection{Codon optimization and silico cloning process of the final candidate}

According to the results of the study, the customized nucleotide sequence of the vaccine construct has a CAI score of 0.972 (codon adaptation index) and is highly expressive in the $\mathrm{E}$. coli strain $\mathrm{K} 12$. The range of $0.8-1.0$ is considered to be a desirable value for the codon adaptation index. Furthermore, the concentration of GC (Guanine-Cytosine) was determined to be $56.05 \%$, which is within the acceptable range of $30-70 \%$ for effective translation, according to the findings. The predicted DNA sequences for the vaccine were cloned into $\mathrm{pET}$ $28 \mathrm{a}(+)$ cloning vector plasmids using the SnapGene program, with EcoRI serving as the beginning site restriction enzyme and BamHI serving as the end site restriction enzyme. The cloned plasmid, which contained the vaccine DNA sequences that had been created, was discovered to be 6575 bp in length (Figure 10). It is indicated in red, along with restriction sites. $6 x$ histidine residues and thrombin are found in this plasmid, which will aid in the purification of the vaccine and its subsequent downstream processing.

\section{Discussion}

Kaposi sarcoma cancer is a cancer that affects people who have a weakened immune system. It is a life-threatening illness that affects adults in particular. The standard retroviral and anticancer drugs, including as chemotherapy, are used to treat KS cancer all over the world, although they are not always effective in treating it. Aside from that, chemotherapy with pegylated liposomal doxorubicin or paclitaxel is the most often used treatment for advanced-stage KS cancer, despite the fact that it is infrequently effective [1]. Due to the fact that Kaposi sarcoma cancer is not similarly expressed in all instances, those various therapeutic approaches provide very limited protection against Kaposi sarcoma cancer in adults, who account for the majority of Kaposi sarcoma cancer cases globally. As a consequence, a new 
candidate vaccine against HHV-8 is urgently required, one that can protect a large proportion of the population while also providing good results against the virus. The advancement of reverse vaccinology, as well as the availability of genomics and proteomics information, aid in the formulation of vaccines. Additionally, as compared to conventional vaccine design, effective adoption of bio-informatics techniques is advantageous [62]. Because it has the potential to be utilized for in-silico epitope prediction the identification of immunogenic antigens is a critical stage in the vaccine design process [63]. Immunogenic antigens are distinguished by their ability to adhere and stimulate immune cells, as well as their ability to function as immuno-dominant antigens [64]. We used computational methods to conduct an organized and comprehensive evaluation of the five surface proteins of virus glycoproteins ( $\mathrm{gB}, \mathrm{gM}, \mathrm{gN}, \mathrm{gH}$, and gL) that make up HHV-8 vaccines. Our study team predicted in total of 18 (CTL, HTL and linear $\mathrm{BCL}$ ) epitopes using bioinformatics approaches, which we think may have the capacity to induce both cellular and humoral response [63]. The construction of multi epitope vaccine relies on the prediction of Human Herpes Virus-8 (B-cell and T-cell) epitopes sequences, which are important in the development of biological and cellular functions as well as it predicted to be stimulating for innate and adaptive immunity in vitro. Vaccine design relies on BCL epitope prediction [65], as it distinguish the antigen-antibody interaction site[66]. According to the findings of this study, a 20-mer linier B-cell epitope was predicted using the BCpred server. It is important for T-cell epitopes to be found in order for adaptive immunity to initiate. The NetCTL and Tepitool servers predicted 9mer CTL and 15-mer HTL epitope that triggered adaptive immunity functions appropriately with MHC molecules [67]. So, finding epitopes that can be cooperated by MHC is very important in predicting which T-cell epitopes are potent [26]. Additionally, identification of CD4+ and CD8+ T cells is crucial for designing vaccines based on multiple epitopes [68].

Finally, our predicted BCL, CTL and HTL epitopes from extracted glycoprotein sequences and linked together with AAY and GGGS linkers [69] to develop an multi-epitope based vaccine. To ability to develop immune response in vaccine sequences, GGGS, GPGPG, and AAY linkers were incorporated between chosen epitopes, allowing for the systematic construction of a candidate vaccine sequence [70]. Additionally, the EAAAK linker joined among the epitope's amino acid sequences and the adjuvants to optimize vaccine production and bioactivity. The MHC Class I, MHC Class II, IFN-y, IL-4, IL-10, and line B cell epitopes were identified by assessing various physicochemical and structural parameters of the produced vaccine. Lower allergenicity and higher antigenicity in the protein vaccine has increased its effectiveness as a potential vaccine. Adjuvants are used to balance the lower immunogenicity in construct [71]. The multi - epitope vaccine design has a molecular weight of $43 \mathrm{kDa}$ and a calculated pl of 9.73, indicating that it is alkaline. The aliphatic index indicates that the protein contains aliphatic side chains, indicating probable hydrophobicity. The score has been assessed to be highly soluble following expression and to have a high immunogenicity. Primarily, the solubility of overexpressed recombinant proteins in the E. coli host is crucial for several biochemical and functional investigations [72].

Then secondary and tertiary structures for the vaccine candidate sequence were predicted. Secondary structure analysis revealed that the protein was composed mostly of $46.52 \%$ a-helices and $39.55 \%$ coils. The unfolded protein domains and a-helical coiled-coil peptides have been suggested to be antigenic in structure. Both structural categories have been demonstrated to fold into the recipient's natural forms, enabling them to be identified by antibodies produced in reaction to infection [73]. The tertiary structure of the vaccine candidate has significantly improved during refining and exhibited suitable properties as determined by the detailed Ramachandran plot data. The Ramachandran plot reveals that $94.8 \%$ of residues originate in preferred areas, while $3.7 \%$ additional allowed areas with less $0.9 \%$ residues generously allowed areas; this implies that the overall model's value is acceptable. Screening for immunoreactivity by serological studies is a critical aspect of validating a potential vaccine [71]. Additionally, to ascertain the vaccine's ability to bind to TLRs expressed on various immune cells, the docking of the candidate vaccine was done with TLR-4. The findings indicated that the developed vaccine binds well to TLR-4 and has a strong probability of inducing both innate and adaptive immune responses.

Molecular dynamics simulations were utilized to further assess the stability and dynamics of the TLR-4 and vaccine docking complex, with the RMSD and RMSF plots reflecting the complex's steady state binding properties [74]. The immune simulation data showed an increase in memory B-lymphocytes and helper cell lines following repeated antigen exposure. A multi-epitope vaccination induced antigen-specific immune responses against KSHV. Other innate immune cells, such as macrophages and dendritic cells, also performed well in our study.

It is necessary at this point to express the recombinant protein in an appropriate host in order to proceed. The E. coli K12 strain expression methods are the favored choice for recombinant protein expression [75]. Both the GC content $56.05 \%$ and the CAl score 0.972 were advantageous for high-level protein expression and optimization in bacteria. The next step is to produce this peptide in a bacterial system and perform the various immunological assays necessary to corroborate the immunoinformatics study findings.

\section{Conclusion}

The causative agent, Human Herpes Virus-8 (HHV-8), causes cancer when a patient's immune system is unable to fight against the virus, particularly immune impaired HIV patients and organ transplant recipients. This illness deteriorates the patients' condition at a time when medication is ineffective. While people suffer from Kaposi sarcoma malignancy, there is now no pharmacological option accessible to treat a 
broad spectrum of KS patients. Novel vaccine candidates that cannot be obtained only via preclinical, in vitro, or animal trials. We used computational methodologies to produce a multi-epitope vaccine against HHV-8 that is highly immunogenic and has the required features for use as a carrier vaccine. Numerous techniques were used rigorously to generate a multi epitope vaccination. The evaluation of population coverage and conservancy, molecular docking, molecular dynamics simulations, and immunity simulations demonstrates that the newly designed vaccine is well accepted and safe in all parameters. Now, our chimeric multi epitope vaccination must be analyzed in the laboratory to see if it is suitable for real-world use in the treatment of Kaposi sarcoma cancer.

\section{Declarations}

\section{Author Contributions}

Sumaiya: Methodology, Experiment Design, Data curation, Manuscript Writing, Molecular Dynamics Simulation

Mahmudul Hasan: Data curation, Results analysis

Md. Mashiur Rahaman: Experiment Design, Manuscript Writing, Molecular Dynamics Simulation

G. M. Nurnabi Azad Jewel: Project Supervision, Formal analysis Data curation

Hammadul Hoque: Conceptualization, Supervision, Formal analysis, and Reviewing

\section{Conflict of Interest}

The authors declare no conflict of interest.

\section{References}

1. Cesarman, E., et al., Kaposi sarcoma. Nature Reviews Disease Primers, 2019. 5(1): p. 9.

2. Barrett, L., et al., Role of Interleukin-1 Family Members and Signaling Pathways in KSHV Pathogenesis. Frontiers in Cellular and Infection Microbiology, 2020. 10: p. 679.

3. Iftode, N., et al., Update on Kaposi sarcoma-associated herpesvirus (KSHV or HHV8)-review. Romanian Journal of Internal Medicine, 2020. 58(4): p. 199-208.

4. Arranz-Caso, A., et al., Kaposi sarcoma presenting shortly after primary infection by HIV and human herpesvirus-8. Aids, 2018. 32(2): p. 271-275.

5. Rewane, A. and P. Tadi, Herpes Virus Type 8. 2020.

6. Adegbidi, H., et al., Epidemic, Endemic, or Stewart-Bluefarb? When Several Forms of Kaposi Seem to Dispute Paternity. Case reports in dermatological medicine, 2020. 2020.

7. Wu, L., et al., Three-dimensional structure of the human herpesvirus 8 capsid. Journal of virology, 2000. 74(20): p. 9646-9654.

8. Yan, L., et al., Towards better understanding of KSHV life cycle: from transcription and posttranscriptional regulations to pathogenesis. Virologica Sinica, 2019. 34(2): p. 135-161.

9. Plancoulaine, S. and A. Gessain, Epidemiological aspects of human herpesvirus 8 infection and of Kaposi's sarcoma. Medecine et maladies infectieuses, 2005. 35(5): p. 314-321.

10. Mbulaiteye, S.M., et al., Human herpesvirus 8 infection and transfusion history in children with sickle-cell disease in Uganda. Journal of the National Cancer Institute, 2003. 95(17): p. 1330-1335.

11. Cesaro, S., et al., Incidence and outcome of Kaposi sarcoma after hematopoietic stem cell transplantation: a retrospective analysis and a review of the literature, on behalf of infectious diseases working party of EBMT. Bone marrow transplantation, 2020. 55(1): p. 110-116.

12. Martín-Carbonero, L., et al., Pegylated liposomal doxorubicin plus highly active antiretroviral therapy versus highly active antiretroviral therapy alone in HIV patients with Kaposi's sarcoma. Aids, 2004. 18(12): p. 1737-1740. 
13. Yarchoan, R., et al., Treatment of AIDS-related Kaposi's sarcoma with interleukin-12: rationale and preliminary evidence of clinical activity. Critical reviews in immunology, 2007. 27(5): p. 401.

14. Bernstein, Z.P., et al., A multicenter Phase II study of the intravenous administration of liposomal tretinoin in patients with acquired immunodeficiency syndrome-associated Kaposi's sarcoma. Cancer: Interdisciplinary International Journal of the American Cancer Society, 2002. 95(12): p. 2555-2561.

15. Luppi, M., et al., Molecular evidence of organ-related transmission of Kaposi sarcoma-associated herpesvirus or human herpesvirus-8 in transplant patients. Blood, The Journal of the American Society of Hematology, 2000. 96(9): p. 3279-3281.

16. Nichols, L.A., L.A. Adang, and D.H. Kedes, Rapamycin blocks production of KSHV/HHV8: insights into the anti-tumor activity of an immunosuppressant drug. PloS one, 2011. 6(1): p. e14535.

17. Versteeg, L., et al., Enlisting the mRNA vaccine platform to combat parasitic infections. Vaccines, 2019. 7(4): p. 122.

18. Fadaka, A.O., et al., Immunoinformatics design of a novel epitope-based vaccine candidate against dengue virus. Scientific Reports, 2021. 11(1): p. 1-22.

19. Bahmani, B., et al., HPV16-E7 Protein T Cell Epitope Prediction and Global Therapeutic Peptide Vaccine Design Based on Human Leukocyte Antigen Frequency: An In-Silico Study. International Journal of Peptide Research and Therapeutics, 2021. 27(1): p. 365-378.

20. Kardani, K., A. Bolhassani, and A. Namvar, An overview of in silico vaccine design against different pathogens and cancer. Expert Review of Vaccines, 2020. 19(8): p. 699-726.

21. Pruitt, K.D., T. Tatusova, and D.R. Maglott, NCBI Reference Sequence (RefSeq): a curated non-redundant sequence database of genomes, transcripts and proteins. Nucleic acids research, 2005. 33(suppl_1): p. D501-D504.

22. Boutet, E., et al., Uniprotkb/swiss-prot, in Plant bioinformatics. 2007, Springer. p. 89-112.

23. Doytchinova, I.A. and D.R. Flower, VaxiJen: a server for prediction of protective antigens, tumour antigens and subunit vaccines. BMC bioinformatics, 2007. 8(1): p. 1-7.

24. Larsen, M.V., et al., Large-scale validation of methods for cytotoxic T-lymphocyte epitope prediction. BMC bioinformatics, 2007. 8(1): p. 1-12.

25. Nielsen, M., et al., Reliable prediction of T-cell epitopes using neural networks with novel sequence representations. Protein Science, 2003. 12(5): p. 1007-1017.

26. Paul, S., et al., TepiTool: a pipeline for computational prediction of T cell epitope candidates. Current protocols in immunology, 2016. 114(1): p. 18.19. 1-18.19. 24.

27. Saha, I., G. Mazzocco, and D. Plewczynski, Consensus classification of human leukocyte antigen class II proteins. Immunogenetics, 2013. 65(2): p. 97-105.

28. EL-Manzalawy, Y., D. Dobbs, and V. Honavar, Predicting linear B-cell epitopes using string kernels. Journal of Molecular Recognition: An Interdisciplinary Journal, 2008. 21(4): p. 243-255.

29. Dimitrov, I., et al., AllerTOP v. 2-a server for in silico prediction of allergens. Journal of molecular modeling, 2014. 20(6): p. 1-6.

30. Gupta, S., et al., Peptide toxicity prediction, in Computational peptidology. 2015, Springer. p. $143-157$.

31. Krogh, A., et al., Predicting transmembrane protein topology with a hidden Markov model: application to complete genomes. Journal of molecular biology, 2001. 305(3): p. 567-580.

32. Wu, J., et al., DeepHLApan: a deep learning approach for neoantigen prediction considering both HLA-peptide binding and immunogenicity. Frontiers in immunology, 2019. 10: p. 2559.

33. Dhanda, S.K., P. Vir, and G.P. Raghava, Designing of interferon-gamma inducing MHC class-/l binders. Biology direct, 2013. 8(1): p. 115. 
34. Pahari, S., et al., Morbid sequences suggest molecular mimicry between microbial peptides and self-antigens: a possibility of inciting autoimmunity. Frontiers in microbiology, 2017. 8: p. 1938.

35. Nagpal, G., et al., Computer-aided designing of immunosuppressive peptides based on IL-10 inducing potential. Scientific reports, 2017. 7(1): p. 1-10.

36. Bui, H.H., et al., Predicting population coverage of T-cell epitope-based diagnostics and vaccines. BMC Bioinformatics, 2006. 7: p. 153.

37. Bui, H.-H., et al., Development of an epitope conservancy analysis tool to facilitate the design of epitope-based diagnostics and vaccines. BMC bioinformatics, 2007. 8(1): p. 1-6.

38. Kim, J., et al., Human $\beta$-defensin 2 plays a regulatory role in innate antiviral immunity and is capable of potentiating the induction of antigen-specific immunity. Virology journal, 2018. 15(1): p. 1-12.

39. Kim, D.T., et al., Introduction of soluble proteins into the MHC class I pathway by conjugation to an HIV tat peptide. The Journal of Immunology, 1997. 159(4): p. 1666-1668.

40. Nezafat, N., et al., A novel multi-epitope peptide vaccine against cancer: an in silico approach. Journal of theoretical biology, 2014. 349: p. 121-134.

41. Safavi, A., et al., In silico analysis of transmembrane protein 31 (TMEM31) antigen to design novel multiepitope peptide and DNA cancer vaccines against melanoma. Molecular immunology, 2019. 112: p. 93-102.

42. Livingston, B., et al., A rational strategy to design multiepitope immunogens based on multiple Th lymphocyte epitopes. The Journal of Immunology, 2002. 168(11): p. 5499-5506.

43. Shanmugam, A., et al., Synthetic Toll like receptor-4 (TLR-4) agonist peptides as a novel class of adjuvants. PLoS One, 2012. 7(2): p. e30839.

44. Hon, J., et al., SoluProt: prediction of soluble protein expression in Escherichia coli. Bioinformatics, 2021. 37(1): p. 23-28.

45. Buchan, D.W. and D.T. Jones, The PSIPRED protein analysis workbench: 20 years on. Nucleic acids research, 2019. 47(W1): p. W402W407.

46. Kim, D.E., D. Chivian, and D. Baker, Protein structure prediction and analysis using the Robetta server. Nucleic acids research, 2004. 32(suppl_2): p. W526-W531.

47. Heo, L., H. Park, and C. Seok, GalaxyRefine: Protein structure refinement driven by side-chain repacking. Nucleic acids research, 2013. 41(W1): p. W384-W388.

48. Dym, O., D. Eisenberg, and T. Yeates, ERRAT. 2012.

49. Laskowski, R., M. MacArthur, and J. Thornton, PROCHECK: validation of protein-structure coordinates. 2006.

50. Wiederstein, M. and M.J. Sippl, ProSA-web: interactive web service for the recognition of errors in three-dimensional structures of proteins. Nucleic acids research, 2007. 35(suppl_2): p. W407-W410.

51. Burley, S.K., et al., Protein Data Bank (PDB): the single global macromolecular structure archive. Protein Crystallography, 2017: p. 627-641.

52. Kozakov, D., et al., The ClusPro web server for protein-protein docking. Nature protocols, 2017. 12(2): p. 255-278.

53. Weng, G., et al., HawkDock: a web server to predict and analyze the protein-protein complex based on computational docking and MM/GBSA. Nucleic acids research, 2019. 47(W1): p. W322-W330.

54. Laskowski, R.A., PDBsum: summaries and analyses of PDB structures. Nucleic acids research, 2001. 29(1): p. $221-222$.

55. Pikkemaat, M.G., et al., Molecular dynamics simulations as a tool for improving protein stability. Protein Engineering, Design and Selection, 2002. 15(3): p. 185-192. 
56. Van Der Spoel, D., et al., GROMACS: fast, flexible, and free. Journal of computational chemistry, 2005. 26(16): p. 1701-1718.

57. Vanommeslaeghe, K., et al., CHARMM general force field: A force field for drug-like molecules compatible with the CHARMM allatom additive biological force fields. Journal of computational chemistry, 2010. 31(4): p. 671-690.

58. Berendsen, H.J., et al., Molecular dynamics with coupling to an external bath. The Journal of chemical physics, 1984. 81(8): p. 36843690.

59. Rapin, N., et al., Computational immunology meets bioinformatics: the use of prediction tools for molecular binding in the simulation of the immune system. PloS one, 2010. 5(4): p. e9862.

60. Faiza, M., et al., In silico multi-epitope vaccine against covid19 showing effective interaction with $H L A-B * 15$ : 03. bioRxiv, 2020.

61. Grote, A., et al., JCat: a novel tool to adapt codon usage of a target gene to its potential expression host. Nucleic acids research, 2005. 33(suppl_2): p. W526-W531.

62. María, R., et al., The impact of bioinformatics on vaccine design and development. Vaccines, 2017. 2: p. 3-6.

63. Yasmin, T., et al., In silico proposition to predict cluster of B-and T-cell epitopes for the usefulness of vaccine design from invasive, virulent and membrane associated proteins of . jejuni. In silico pharmacology, 2016. 4(1): p. 1-10.

64. Galluzzi, L., et al., Immunogenic cell death in cancer and infectious disease. Nature Reviews Immunology, 2017. 17(2): p. 97-111.

65. Galanis, K.A., et al., Linear B-cell epitope prediction for in silico vaccine design: a performance review of methods available via command-line interface. International journal of molecular sciences, 2021. 22(6): p. 3210.

66. Lu, L.L., et al., Beyond binding: antibody effector functions in infectious diseases. Nature Reviews Immunology, 2018. 18(1): p. 4661.

67. Brusic, V., V.B. Bajic, and N. Petrovsky, Computational methods for prediction of T-cell epitopes-a framework for modelling, testing, and applications. Methods, 2004. 34(4): p. 436-443.

68. Lim, H.X., et al., Development of multi-epitope peptide-based vaccines against SARS-CoV-2. Biomedical Journal, 2021. 44(1): p. 1830.

69. Rahmani, A., et al., Development of a conserved chimeric vaccine based on helper T-cell and CTL epitopes for induction of strong immune response against Schistosoma mansoni using immunoinformatics approaches. International journal of biological macromolecules, 2019. 141: p. 125-136.

70. Yadav, S., et al., Design of a multi-epitope subunit vaccine for immune-protection against Leishmania parasite. Pathogens and global health, 2020. 114(8): p. 471-481.

71. Meza, B., et al., A novel design of a multi-antigenic, multistage and multi-epitope vaccine against Helicobacter pylori: an in silico approach. Infection, Genetics and Evolution, 2017. 49: p. 309-317.

72. Khatoon, N., R.K. Pandey, and V.K. Prajapati, Exploring Leishmania secretory proteins to design B and T cell multi-epitope subunit vaccine using immunoinformatics approach. Scientific reports, 2017. 7(1): p. 1-12.

73. Sela-Culang, I., V. Kunik, and Y. Ofran, The structural basis of antibody-antigen recognition. Frontiers in immunology, 2013.4 : p. 302.

74. Ahmad, S.S., et al., Study of Caspase 8 inhibition for the management of Alzheimer's disease: a molecular docking and dynamics simulation. Molecules, 2020. 25(9): p. 2071.

75. Choi, J.H., K.C. Keum, and S.Y. Lee, Production of recombinant proteins by high cell density culture of Escherichia coli. Chemical engineering science, 2006. 61(3): p. 876-885.

\section{Figures}




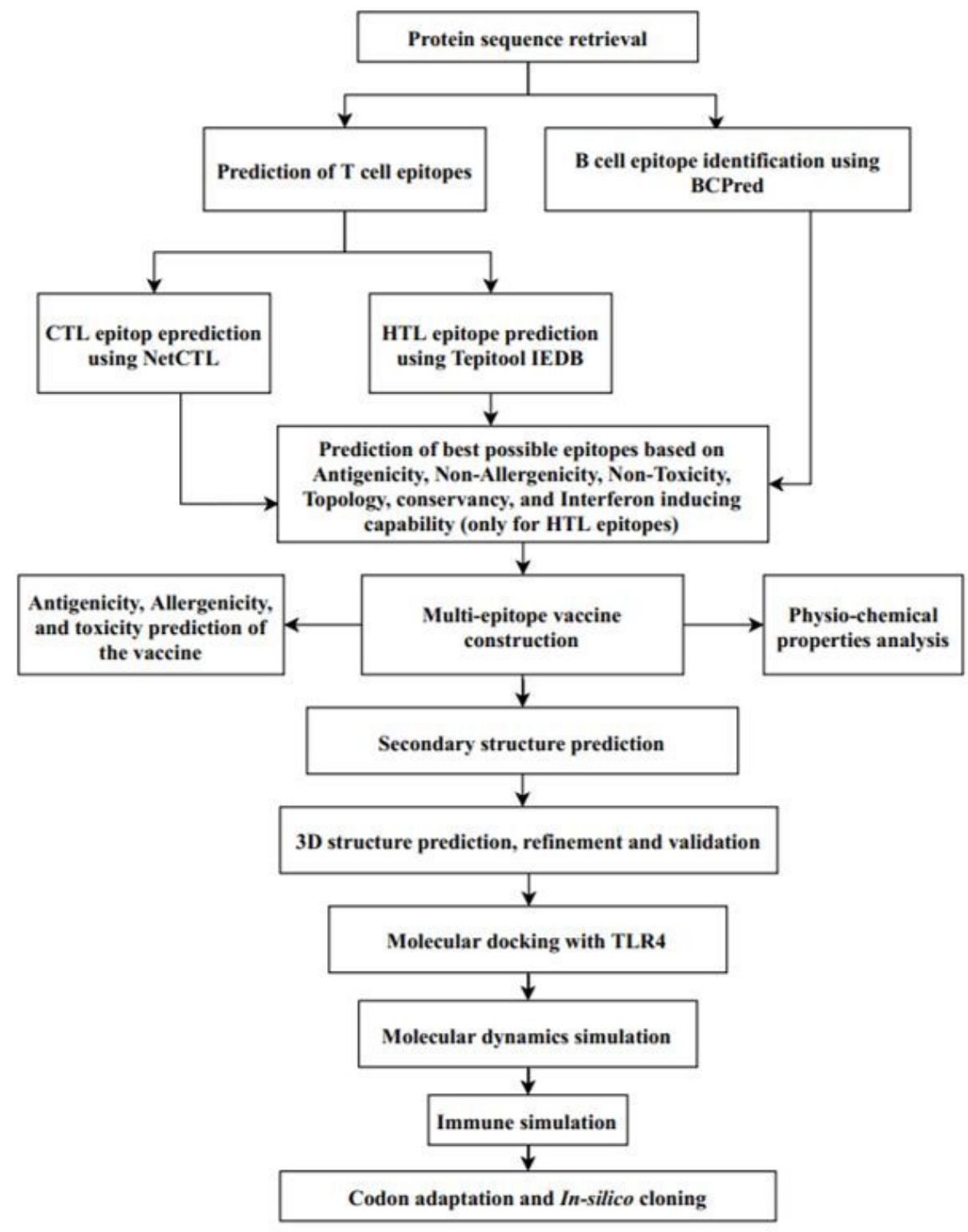

Figure 1

Process flow diagram 


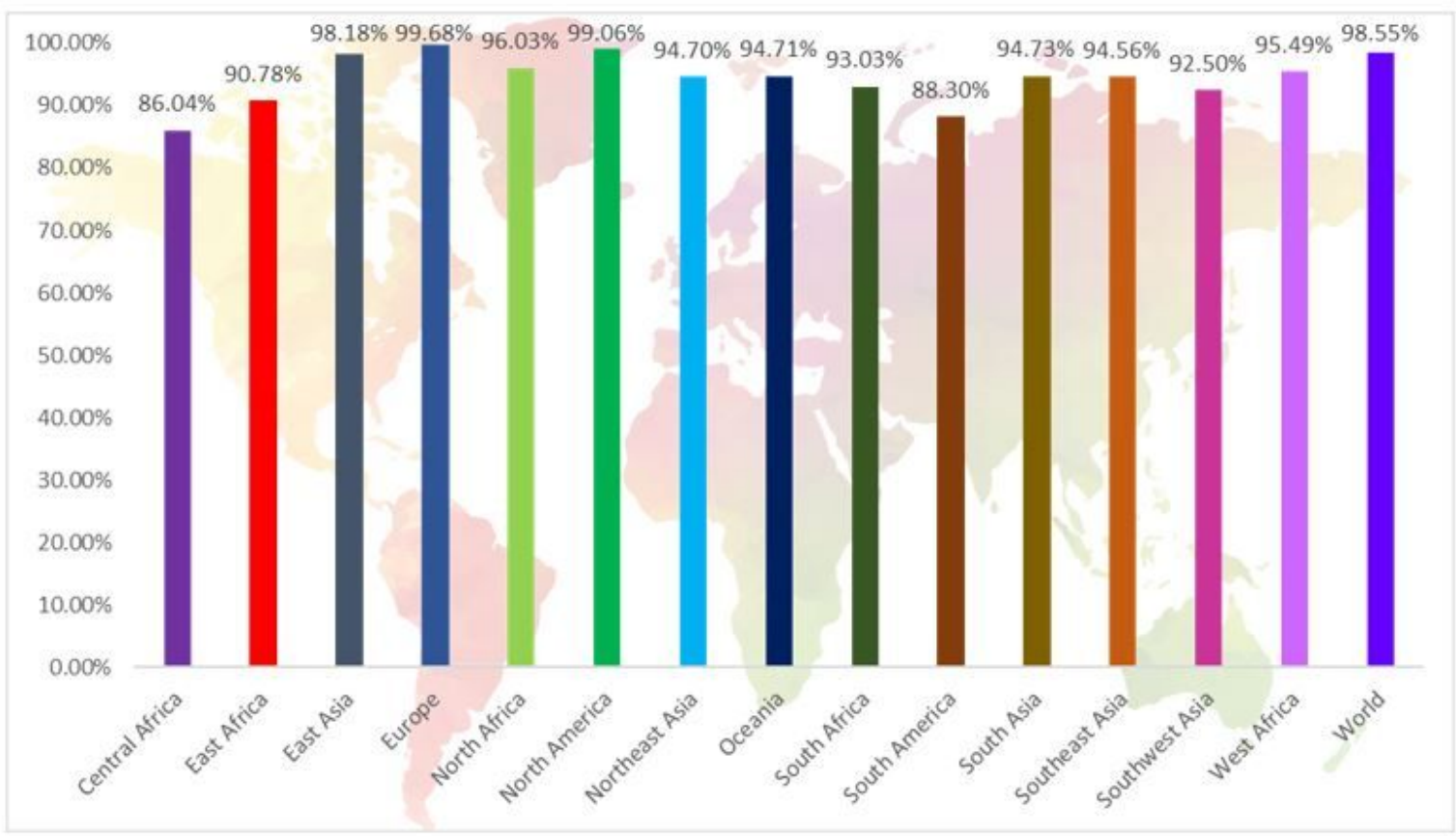

Figure 2

Global coverage of selected CTL epitopes

MEAAAKRKKRRQRRRGGGSGIINTLQKYYCRVRGGRCAVLSCLPKEEQIGKCSTRGRKCCR RKKEAAAKAKFVAAWTLKAAAGGGSIVIAIILIIAAYLATLGTVILAAYMPTFTTVGYAAYVPLP HVTYIAAYFLVAVVRRLAAYFAVHSIHFAAAYKLLFVAVIMAAYLALTVVWYIAAYATFFYLVYL AAYTASTVALALHEYGAEALERAGKHVLMAAFVYCIHFCGPGPGLPLFFAVHSIHFADPGPGP GFLGLRYDLHPAKLRAGPGPGIYRAFSPCFLGLRYDGPGPGAQEAAPDIFLVLFQMGPGPGIL LVCFCAGAAHSRGGPGPGAEPGWFPGIYRVRTTKKSARPSPGPTSVTTPGFYDVAEAAAKAPP HALSHEYGAEALERAGHHHHHH

\section{Figure 3}

An overview of the vaccine candidate sequence having $10 \mathrm{CTL}, 7 \mathrm{HTL}$, and $1 \mathrm{BCL}$ epitopes, with an EAAAK linker attached to the N-terminal Methionine. The AAY, GPGPG, and KK linkers were connected to the $C T L, H T L$, and BCL epitopes respectively. 


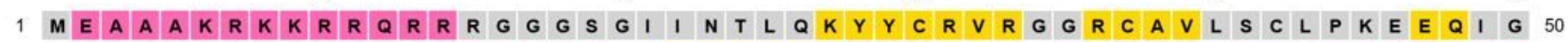

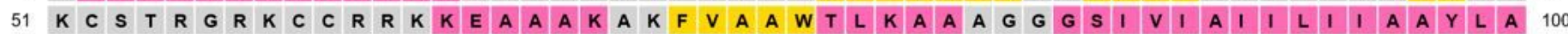

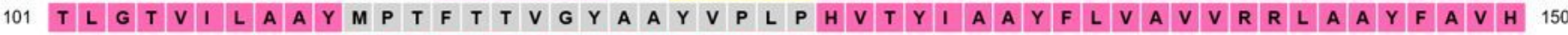

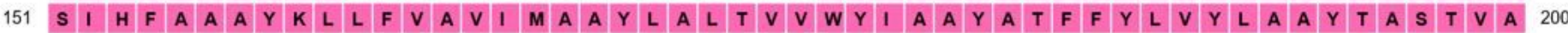

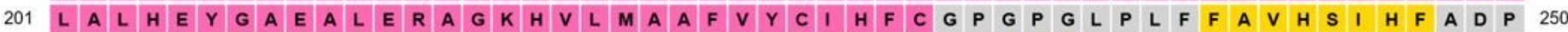

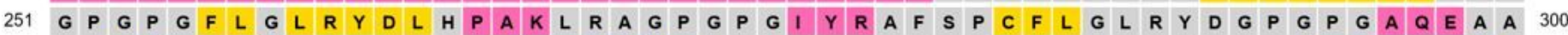

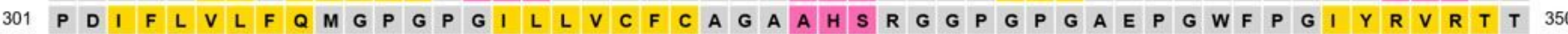

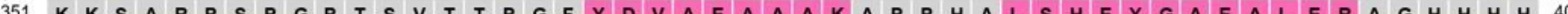
401 H H 20

$$
\text { Helix }
$$

Putative Domain Boundary

Re-entrant Helix
Coil

Membrane Interaction

Cytoplasmic

\section{Disordered}

Transmembrane Helix

Signal Peptide

\section{Figure 4}

Graphical illustration of the secondary structural characterization of the multi-epitope vaccine. The anticipated protein has $46.52 \%$ alpha helical formation, $13.93 \%$ beta-strand, and $39.55 \%$ coil structure. 


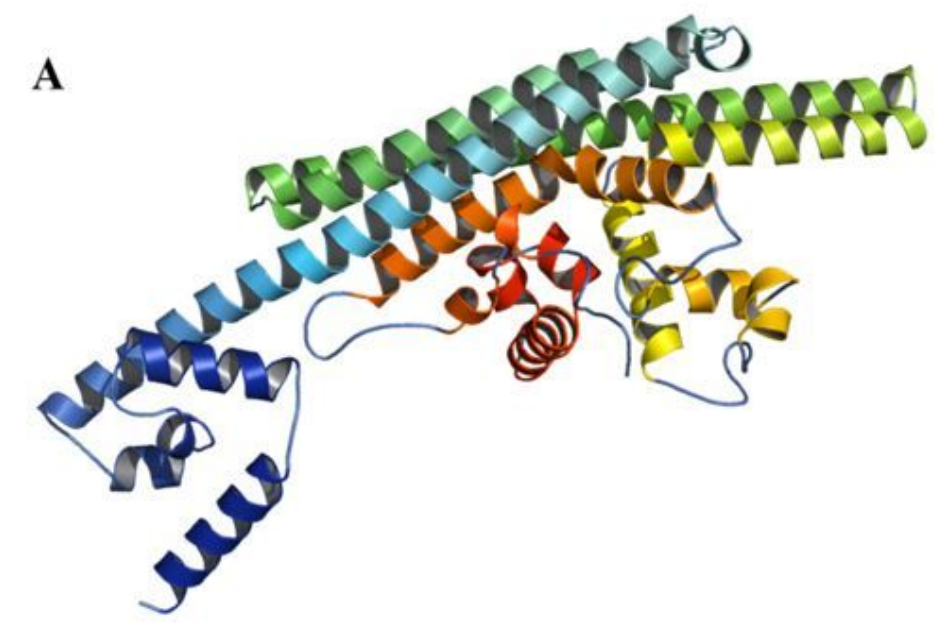

B

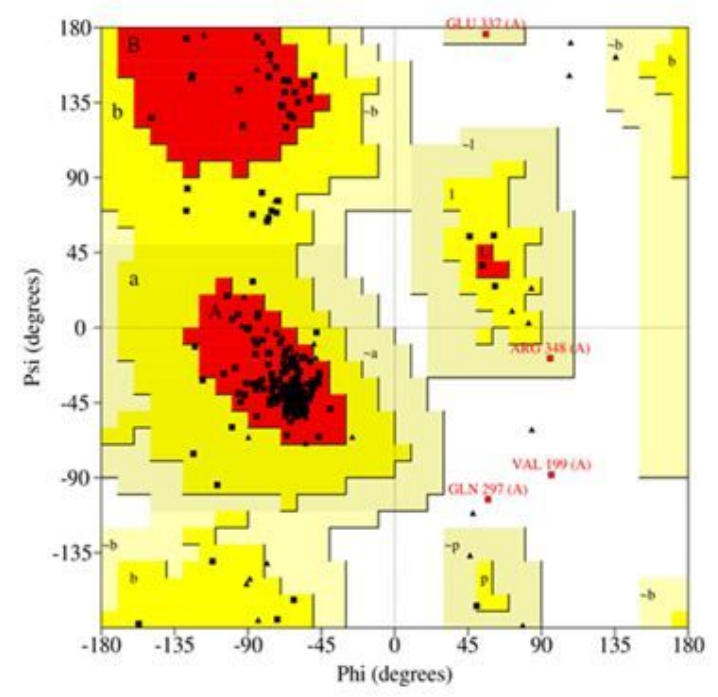

C

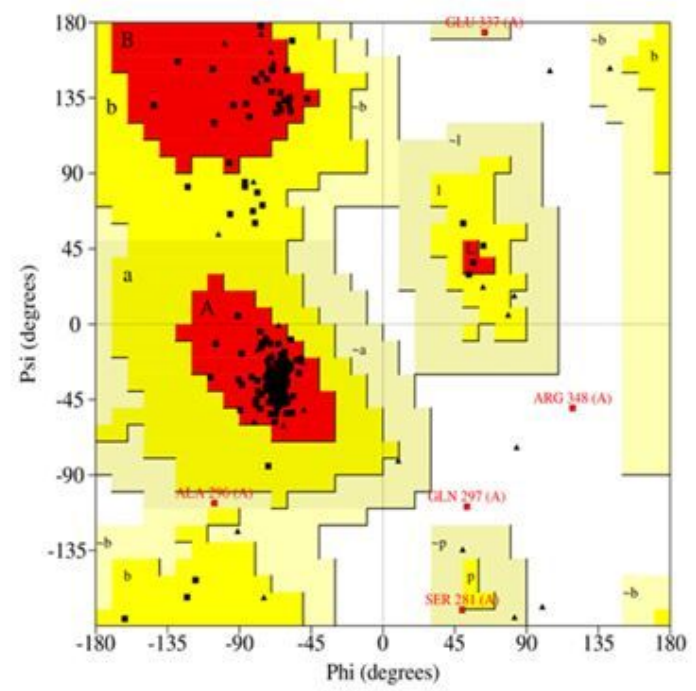

\section{Figure 5}

(A) Refined Tertiary structure of the designated vaccine. Ramachandran plot of the initial modelled vaccine (B) and after refinement (C). 


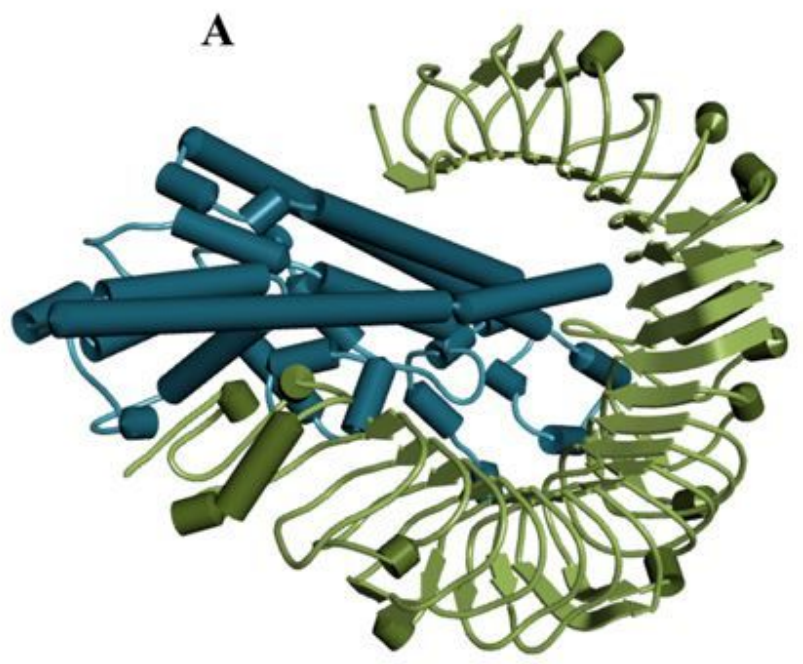

B

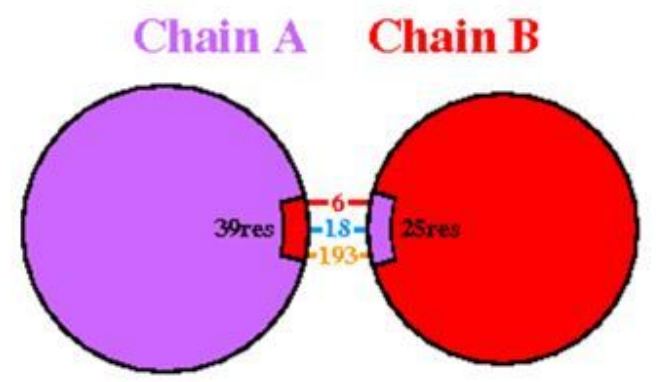

Key: $-\begin{aligned} & \text { Salt } \\ & \text { bridges }\end{aligned}-\begin{aligned} & \text { Disulphide } \\ & \text { bonds }\end{aligned}-\begin{aligned} & \text { Hydrogen } \\ & \text { bonds }\end{aligned} \quad \begin{aligned} & \text { Non-bonded } \\ & \text { contacts }\end{aligned}$

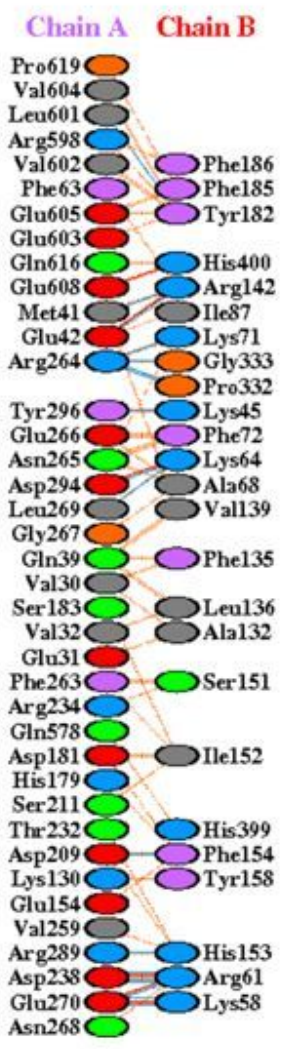

Figure 6

Analyses of the docked complex (A) 3D structure (B) Interface statistics synopsis (C) Bond interaction between TLR4 (chain-A) receptor residues and vaccine (chain-B) residues 


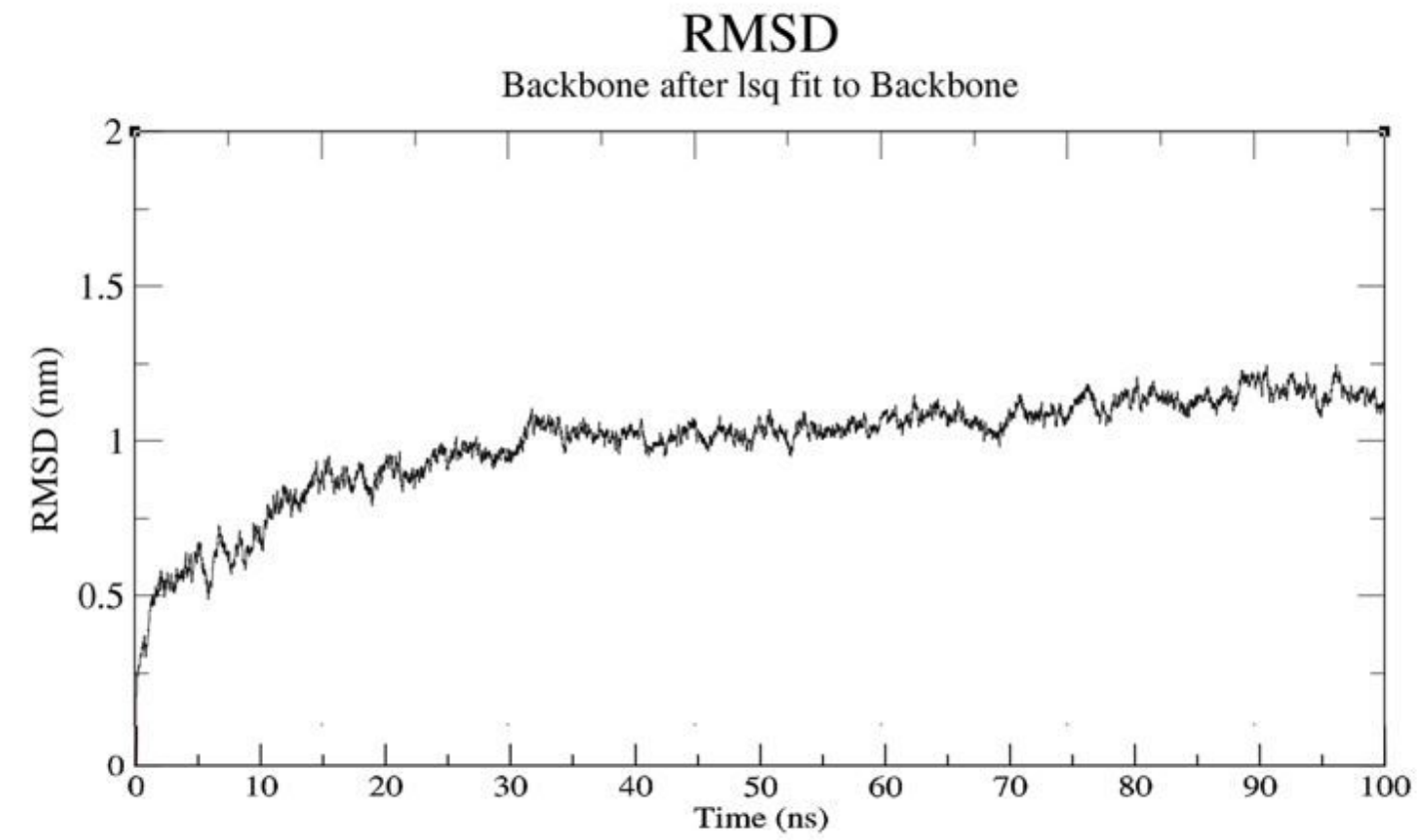

Figure 7

RMSD plot from dynamics simulation showing the structural stability

RMS fluctuation

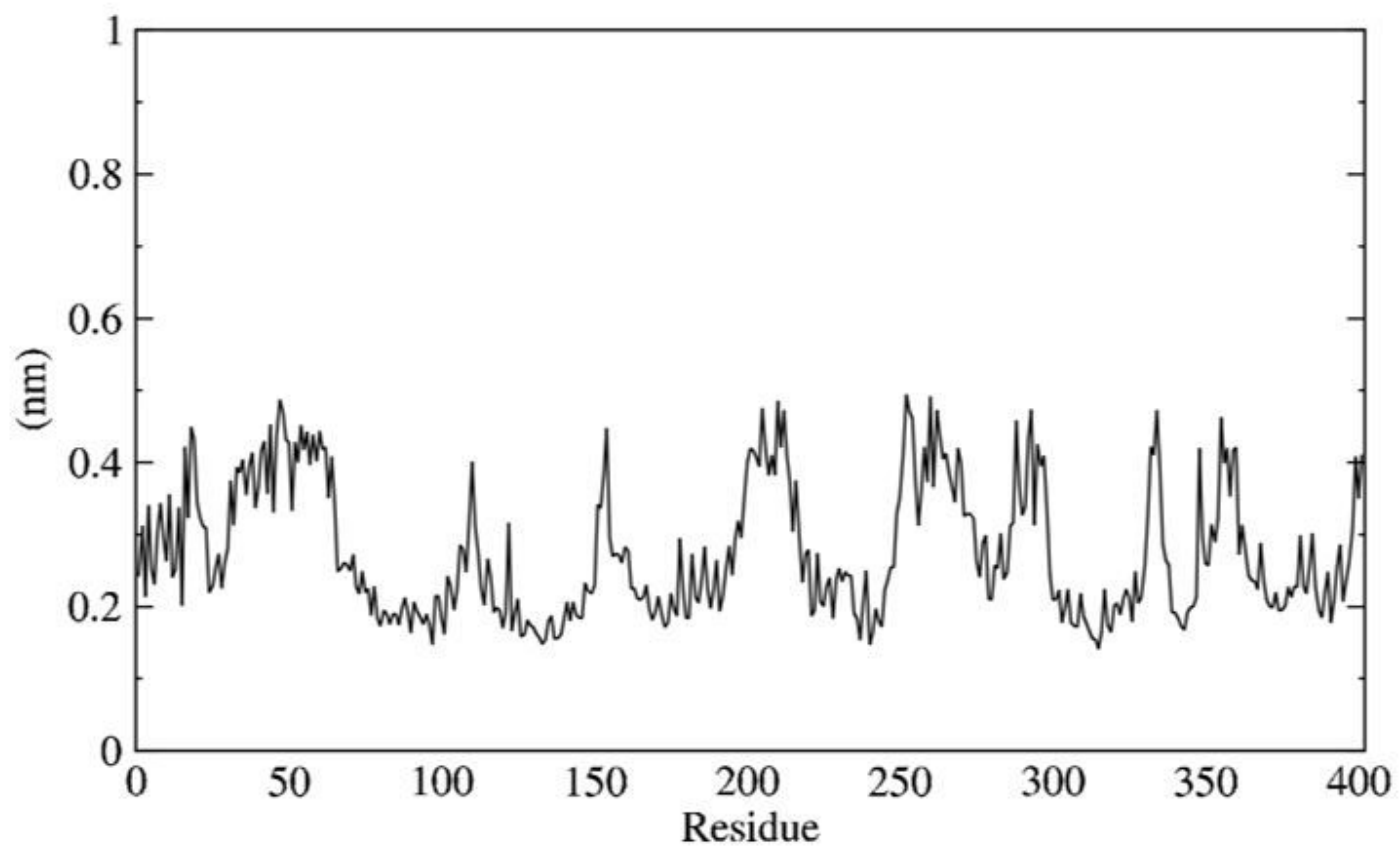

Figure 8

The RMSF plot exhibiting no extreme fluctuations

Page $19 / 21$ 
A Innate Immune Response
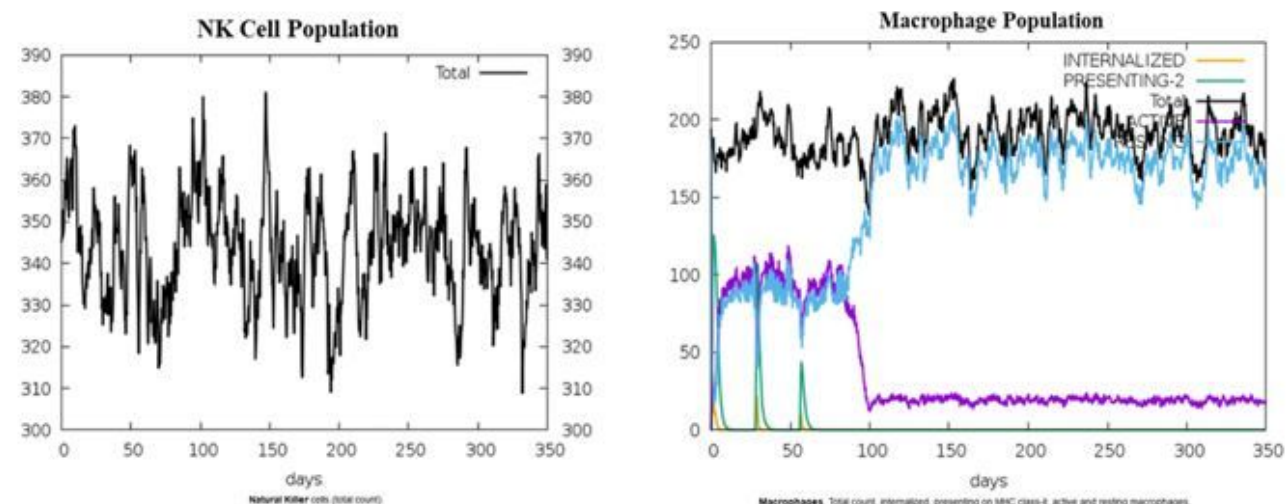

B

Adaptive Immune Response
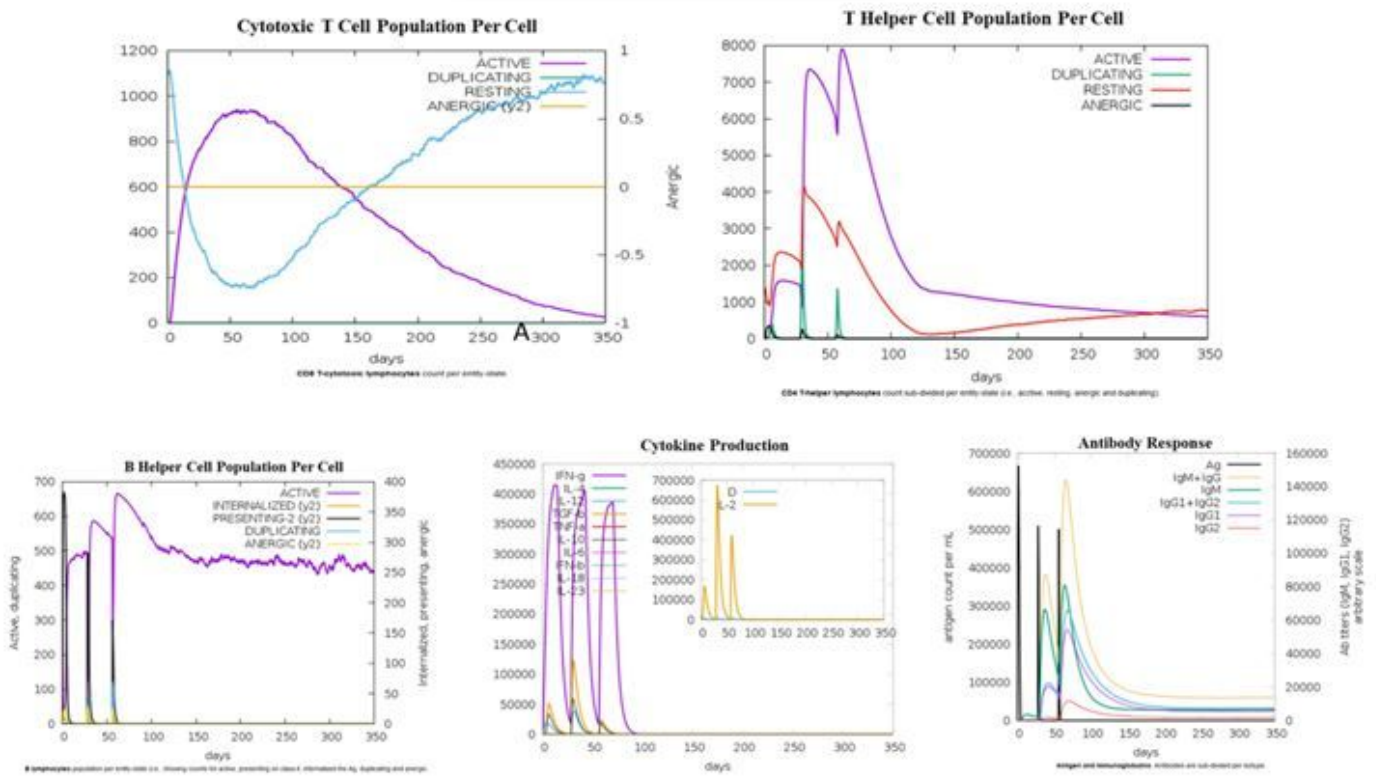

Figure 9

Vaccine immune response simulations. (A) NK cells and macrophages were adequately activated. (B) C-ImmSim server showed an adaptive immune response comprising cytotoxic $T$ cell, $T$ helper, $B$ cell, and antibody responses as well as cytokine generation following vaccination doses. 


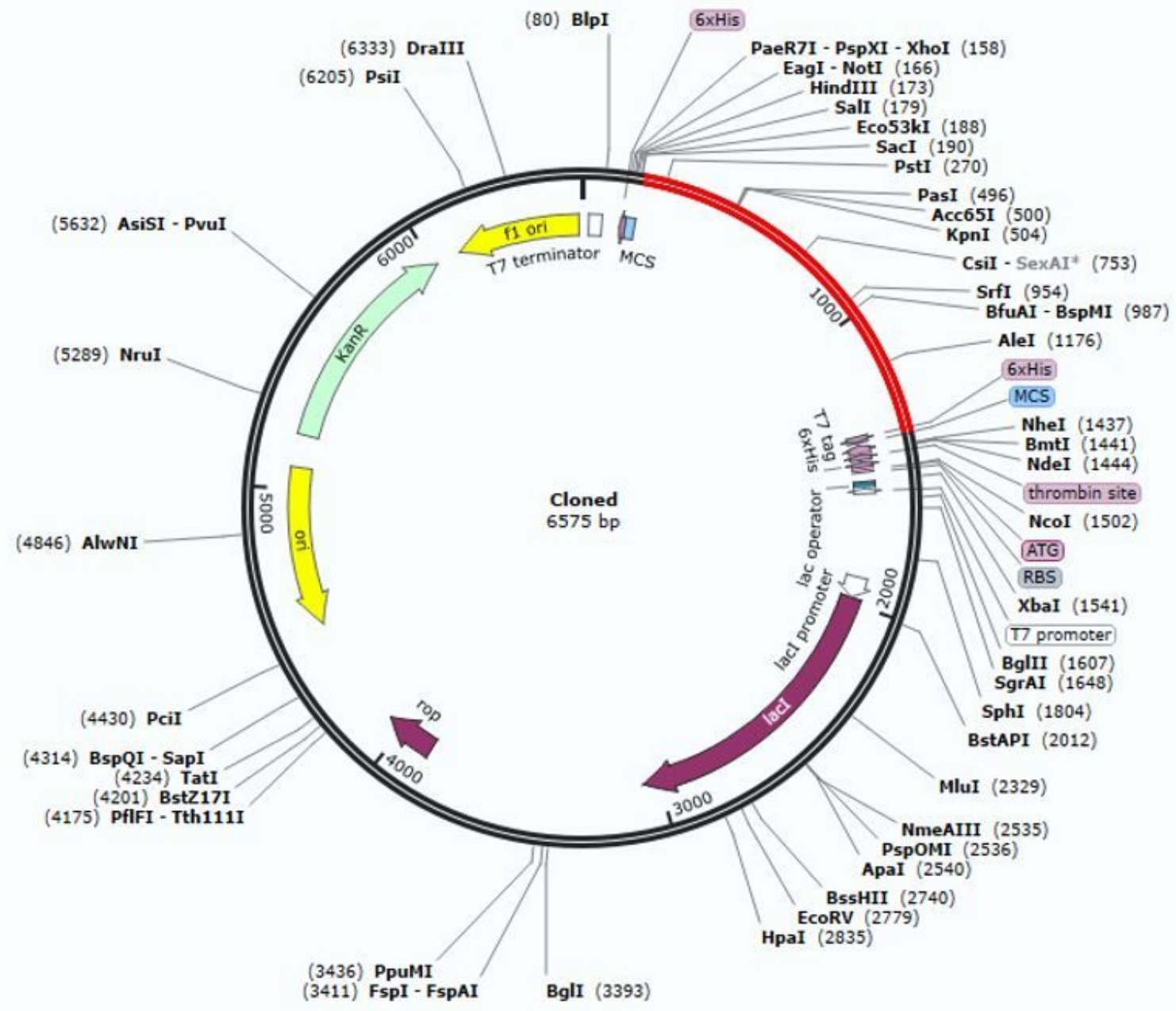

Figure 10

The vaccine nucleotide sequences were cloned into the pET28a (+) expression vector using EcoRI (192 bp) and BamHI (1398 bp) as start and end sites. Red region indicate gene product of the vaccine and black indicates the rest of the plasmid. 